\title{
Umdenken beim Umverteilen
}

\author{
Peter Eichhorn
}

\begin{abstract}
Ein bedingungsloses Grundeinkommen für alle und die Konzentration des Steuereinzugs auf die Mehrwertsteuer - das sind die Gedanken, die derzeit sozialpolitisch die Runde machen. Ein Vorschlag mit Schwächen.
\end{abstract}

Die Vision: Jeder Bürger - ob jung oder alt, in Beruf oder Rente, reich oder arm - erhält ein Grundeinkommen ohne jegliche Bedingung. Derzeit und anfänglich läge dieses garantierte Mindesteinkommen bei monatlich 800 Euro und später bei 1.500 Euro heutiger Kaufkraft. Dieses geschätzte Existenzminimum würde weder durch Steuern noch durch Sozialabgaben geschmälert. An die Stelle der Lohn- und Einkommensteuern träte nämlich eine Ausgaben- bzw. Konsumsteuer etwa in der Art einer Mehrwertsteuer, die der Letztverbraucher aufbrächte (weil ihm kein Vorsteuerabzug möglich ist). Zum Kulturminimum kämen je nach (festgestellter gesundheitlicher) Bedürftigkeit gestaffelte staatliche soziale Leistungen hinzu.

Verfasser dieser Vision ist kein Utopist oder Sozialromantiker, sondern ein erfolgreicher Einzelhändler und Mitgesellschafter der »dm«-Drogeriemarktkette. Einem Unternehmer gleich hat er eine Mission. Sie bezieht sich aber nicht auf ein Produkt oder eine Marke. Seine Botschaft lautet: Nicht bezahlte Arbeit ist höchstes gesellschaftliches Ziel, sondern ein Leben in Freiheit und Würde. Wie man dort hingelangt, ist Gegenstand des Buches »Einkommen für alle«. Es zeichnet sich aus durch einen Paradigmenwechsel und provozierende Thesen, gewürzt mit teils kritischen, teils konstruktiven Beispielen aus der Praxis.

Ohne radikales Umdenken ließen sich Arbeitslosigkeit und gesellschaft- liche Teilnahmslosigkeit sowie Armut auf Dauer nicht verhindern. Der Autor will den Einzelnen finanziell so ausstatten, dass er auf keinen Arbeitsplatz, lies Einkommensplatz angewiesen ist; es ihm vielmehr freisteht, sich beruflich oder sonst wie zu verwirklichen, das heißt mit seinen Talenten in Wirtschaft und Gesellschaft zu wuchern. Auf diesem neutestamentlichen Bild und weiteren theologischen und anthroposophischen Einlassungen, wohl besser: Einstellungen, beruhen viele Passagen. $\mathrm{Zu}$ sätzlich werden ökonomische Argumente herangezogen.

Mit den hier zitierten Sätzen (vgl. Kasten Seite 28) ist der Leser bis zur Hälfte des leicht lesbaren Buches vorgedrungen. Die bisherigen Aussagen gründen auf einer Art Anamnese und Diagnose der Fehleinschätzungen in unserem Arbeitsleben. Es folgen zwei Therapien: Die betriebswirtschaftliche setzt am Beispiel der Drogeriemarktkette »dm « auf Initiative und Eigenverantwortung. Jeder Mitarbeiter soll wie ein Unternehmer denken und bei jeder Investition die Rationalisierung und Kostensenkung erkennen. Führen im Unternehmen bedeutet nicht Menschen zu führen, sondern Bewusstsein zu wecken.

Die volkswirtschaftliche Heilbehandlung fordert einen radikalen Umbau der Besteuerung; aber nicht im Paul Kirchoff'schen Sinne einer pauschalen Einkommensteuer. Götz W. Werner vertritt die Auffassung, dass nur eine Ausgaben-, genauer: eine reine Konsumsteuer wirtschaftlich notwendig und sozial gerecht ist. Jede andere Steuer schaffe nur Scheingerechtigkeiten - ganz zu schweigen von falschen steuerlichen Anreizen, Steuerschlupflöchern, ausufernder Steuerberatung und Ämterbürokratie. Gehe man den Zusammenhängen auf den Grund, müsse

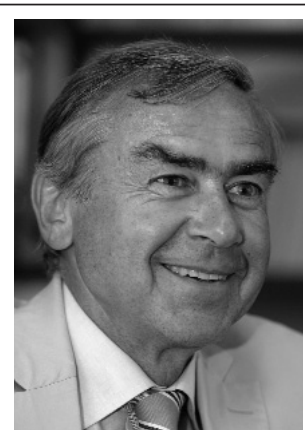

\author{
Prof. Dr. \\ Dr.h.c. \\ mult. Pe- \\ ter Eich- \\ horn ist \\ seit 1981 \\ Ordinarius \\ für Be- \\ triebswirt- \\ schaftsleh-
}

re und Inhaber des Lehrstuhls für Allgemeine Betriebswirtschaftslehre, Public \& Nonprofit Management an der Universität Mannheim. Nach dem Studium der Wirtschaftswissenschaften an den Universitäten Würzburg, FU Berlin und Erlangen-Nürnberg verbrachte er ein Forschungsjahr an der Harvard Business School in Boston und an der University of California in Berkeley. Mit 32 Jahren habilitierte er sich 1972 an der Universität Münster. Er erhielt Rufe an die FU Berlin 1972, die Hochschule für Verwaltungswissenschaften Speyer 1972-78, deren Rektor er 1976/77 war, an die Universität Linz und an die Universität Erlangen-Nürnberg, wo er von 1978 bis 1981 lehrte.

E-Mailoebwl@bwl.uni-mannheim.de

man feststellen, dass es die Aufgabe von Unternehmen ist, Realeinkommen und das heißt konsumierbare Güter oder nachgefragte Dienstleistungen zu produzieren - und nicht Gewinne. Alle Wirtschaftsprozesse enden »immer irgendwo als Einkommen«. Und dies wird »früher oder später immer in reale Güter getauscht - es wird privat konsumiert«. Ferner gelte, dass Unternehmen gar keine Steuern zahlen, denn alle Steuern stecken am Ende in den Preisen und die werden von den Kunden bezahlt. So gesehen gibt es bereits die Konsumsteuer; sie wird nur durch das bisherige Einkommensteuersystem verschleiert. Es käme darauf an, eine Konsum- alias Mehrwertsteuer am Ende der Wertschöpfungskette beim Letztverbraucher auszuweisen und 


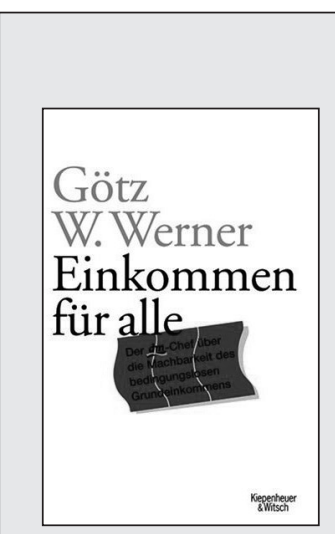

\section{„Einkommen für alle»}

Einkommen ist ein Bürgerrecht, Vollbeschäftigung eine Illusion. Also müssen wir Arbeit und Einkommen trennen. Das ist die Botschaft des Unternehmers Götz W. Werner. Zitate aus seinem Buch »Einkommen für alle«:

»Die im Grundgesetz verankerte Gleichheit und Freiheit aller steht nur auf dem Papier ... Wir alle könnten ... mit dem Grundeinkommen >Lebensunternehmer`, neudeutsch >Freelancerı, sein. ... Solange wir jedoch den Arbeitsbegriff in unseren Köpfen auf den einer bezahlten, weisungsgebundenen, sozialversicherungspflichtigen Vollzeitarbeit beschränken, werfen wir zwei von drei Bürgern aus unserer volkswirtschaftlichen Gesamtbetrachtung menschlicher Arbeit heraus ... Erkenntnis, dass unsere Wirtschaft heute einen Entwicklungsstand erreicht hat, der die Trennung von Arbeit und Einkommen denkbar und möglich macht ...

Armut ist ein finanzielles, kein materielles Problem. Armut ist eine Frage der Verteilung, Mangel dagegen eine Frage der Hervorbringung gesellschaftlichen Reichtums ... Auf dem Felde des Sozialen und Kulturellen wird immer Arbeit im Übermaß gefragt sein. Wir müssen sie uns bloß leisten wollen ...

Geld an sich ist zunächst nur eine nominelle Größe. Vom ärmsten Schuldner bis zum Multimilliardär hat jeder Mensch nur etwas von seinem Nominaleinkommen, wenn er es früher oder später in Realeinkommen verwandelt, es also konsumiert ... Weil wir aber grundsätzlich Einkommen an Arbeit knüpfen, machen wir zugleich einen viel dramatischeren Fehler: Wir halten nur Erwerbsarbeit für Arbeit. Das ist der allergröbste Unfug ... Tatsächlich gibt es sehr viel mehr Arbeit als Erwerbsarbeit. Neben der eigentlichen >Hausarbeit< sind das etwa Erziehung, Pflege, soziales Engagement, Kulturarbeit, Jugendarbeit, Sport, Brauchtum. In all diesen Bereichen werden immense und völlig unverzichtbare Beiträge zum Funktionieren und Gedeihen unserer Gesellschaft geleistet, die ohne diese Arbeit unter unseren Augen zerfallen würde ... Wir reden immer davon, dass jemand einen - oder eben keinen - Arbeitsplatz habe. Dabei haben die meisten Menschen bloß einen Einkommensplatz. Das heißt, sie machen ihre Arbeit einzig und alleine deshalb, weil sie ein Einkommen brauchen. Aber dieses Einkommen brauchten sie ... ohnehin, auch wenn sie keine Arbeit hätten ...

Auf einem freien Markt herrscht das freie Spiel der Kräfte, Angebot und Nachfrage kommunizieren ... Der >Arbeitsmarkt < ist de facto überhaupt kein Markt im Sinne der Marktwirtschaftslehre, denn die einen können, die anderen müssen arbeiten. Von einem freien Spiel der Kräfte und von der Möglichkeit der Wahl kann keine Rede sein. Einen echten Arbeitsmarkt müsste folgender Grundsatz regeln: Alle können, aber keiner muss arbeiten! ... Das Erstaunlichste ... ist ..., dass niemand von der Pflicht ... zur Arbeit spricht, sondern ganz feierlich vom >Recht auf Arbeit « ... Aus dem Zwang wird also flugs ein Recht geschmiedet, das ... in der praktizierten Form nichts anderes bedeutet als eine reale Entrechtung. Nämlich eine wesentliche Einschränkung des ersten und elementarsten Grundrechtes auf Freiheit ... Wir brauchen ein Recht auf Einkommen. Genauer gesagt, auf ein bedingungsloses Grundeinkommen ...

Wir leben hundert Prozent in der Fremdversorgung, wir alle stellen ... nichts mehr selbst her, das uns das Überleben aus eigener Kraft sichern könnte. Selbst >Bauern ‘... sind ja heute keine Selbstversorger mehr ... Einkommen muss in der Fremdversorgungswirtschaft ... gesichert sein ... Einkommen, das die gesellschaftliche Teilhabe erst möglich macht ... Wenn der Bürger ein Einkommen bekommt, um frei von den dringlichsten Existenzsorgen zu sein, ist er frei, etwas Sinnvolles zu tun. Was jeder aus seinem Leben jenseits der Grundversorgung macht, liegt dann vor allem, in eigener Verantwortung ... Fabrikbesitzer ... ihr eigentliches Ziel ist es, mit der gleichen Menge eingesetzter menschlicher Arbeit eine immer größere Menge von Gütern zu produzieren (oder die gleiche Menge von Gütern mit immer weniger menschlicher Arbeit) ... für die Arbeit am Menschen, für die Kulturarbeit gilt dagegen ... das genaue Gegenteil. So lässt sich die Produktivität der Arbeit am Menschen nicht im gleichen Sinne permanent steigern ... im Bildungswesen ... geht es ... eher darum, wie viele Schüler eine Schulform, eine Schule oder meinetwegen auch ein Lehrer zu einem möglichst guten Abschluss führt ...

... in der alten Arbeit Einkommensmaximierung ... Bei der neuen Arbeit ... Sinnmaximierung ... Die Produktivitätsgewinne der alten Arbeit sind die Kostennachteile der neuen Arbeit. Deshalb haben wir den Eindruck, wir könnten uns solche Arbeiten `nicht mehr leisten<. Dabei können wir sie bloß nicht bezahlen. Was wir dagegen sehr gut könnten, ist solche Arbeit zu ermöglichen ... Grundeinkommen ... ermöglichen Arbeit, die man nicht bezahlen kann ...

Wenn das Grundeinkommen eingeführt werden soll, dann muss sich langfristig auch unser Steuersystem von Grund auf ändern ... Fallen alle Steuern bis auf die Konsumsteuer weg, könnte man das Grundeinkommen ansehen ... auch als einen bar ausgezahlten Steuerfreibetrag. Das bedingungslose Grundeinkommen muss als Teil des gesamten Einkommens eines jeden Bürgers verstanden werden. Es ist so etwas wie ein Sockel, der durch Einkünfte aus Erwerbstätigkeit oder unternehmerischer Initiative beliebig aufgestockt werden kann ... Das Grundeinkommen will ... den Zwang, einer schlecht bezahlten und zudem unbefriedigenden Arbeit nachzugehen, beseitigen und damit den Menschen die Möglichkeit einräumen, bei Sicherung ihrer existenziellen Grundbedürfnisse einer sinnvollen Arbeit nachzugehen ...« 
einzubehalten. Selbst im Verhältnis zum Ausland hätte dies Vorteile, denn die Abkehr von Einkommenund Unternehmenssteuern und die Hinwendung zur Konsumsteuer hätte zur Folge, dass viele Leistungen einerseits wieder in Deutschland erbracht, andererseits wesentlich preisgünstiger exportiert werden könnten.

Die Quintessenz für den Leser: Es ist höchste Zeit, dass wir Menschen erstens Arbeit als Befreiung und Arbeitslosigkeit als Belastung, zweitens ein Grundeinkommen als Existenzbzw. Kulturminimum und als Ausgangspunkt für Erwerbs- und Kulturarbeit begreifen. Drittens sollten Initiativen und Verantwortung unser Handeln bestimmen und viertens gilt es das Steuersystem dementsprechend $\mathrm{zu}$ revolutionieren.

Die Frage bleibt, ob solche Ideen als illusionäre Weltverbesserung apostrophiert oder als reale Chance verstanden werden, um Fehlentwicklungen im Berufs- und Gesellschaftsleben zu stoppen. Eine Antwort darauf ist vom Ergebnis mehrerer Wirkungsanalysen abhängig. Kann man davon ausgehen, dass die Meinungsführer und mündigen Bürger bereit und fähig sind, Grundeinkommen und Kulturarbeit zu akzeptieren? Wie wird sich der gesteigerte Mehrwertsteuersatz (etwa bis zur Höhe der Staatsquote von annährend 50 Prozent) beim Endverbraucher auf Preisniveau, Konsum, Außenhandel und Schwarzarbeit auswirken?

Da ein nationaler Alleingang irreal sein dürfte, müssten EU-weite Anpassungen vorgenommen und evaluiert werden. Überhaupt wären die Entwicklungsschritte der Steuersubstitution im In- und Ausland zu definieren und durchzusetzen. Schließlich wären die Volumina zu berechnen sowohl für das benötigte Grundeinkommen für Hartz-IV-Empfänger und weitere Niedriglohnempfänger als auch für zusätzliche bereitzustellende Sozialleistungen nach zu bestimmenden Bedürftigkeitslagen.

Man sieht: Für eine zweite Auflage des Buches gibt es noch viel zu tun.

\section{Themenhefte der Blätter der Wohlfahrtspflege}

Der Allgemeine Sozialdienst

Blätter der Woblfahrtspflege 1/2002

Lebensführung, Lebensbewältigung

Blätter der Woblfahrtspflege 2/2002

Daseinsvorsorge

Extra: Die PISA-Studie und die

Bürgergesellschaft

Blätter der Woblfahrtspflege 3/2002

Armut und Integration

Blätter der Wohlfahrtspflege 4/2002

Lebenslagen im Alter

Blätter der Woblfahrtspflege 5/2002

Bürgerbeteiligung

Blätter der Woblfahrtspflege 6/2002

Bildung und Soziale Arbeit

Blätter der Woblfahrtspflege 1/2003

Menschen mit Behinderungen

Blätter der Woblfahrtspflege 2/2003

Familienpolitik, Familienhilfe

Blätter der Wohlfahrtspflege 3/2003

Fachsozialarbeit

Blätter der Woblfahrtspflege 4/2003

Unternehmensführung in der Altenhilfe Blätter der Woblfahrtspflege 5/2003

Elementarerziehung

Blätter der Woblfahrtspflege 6/2003

Kommunale Sozialpolitik

Blätter der Woblfahrtspflege 1/2004

Case Management

Blätter der Woblfahrtspflege 2/2004

Brennpunkte der Jugendhilfe

Blätter der Woblfahrtspflege 3/2004

Ambulante Pflege

Blätter der Woblfahrtspflege 5/2004

Interkulturelle Arbeit

Blätter der Woblfahrtspflege 6/2004

Demografischer Wandel

Blätter der Woblfahrtspflege 1/2005

Bildung

Blätter der Woblfahrtspflege 2/2005

Bürgerengagement

Blätter der Wohlfahrtspflege 3/2005

Gemeindepsychiatrie

Blätter der Woblfahrtspflege 4/2005

Standards Sozialer Arbeit

Blätter der Woblfahrtspflege 5/2005
Performanz - Wissen, was man tut, und zeigen, was man leistet Blätter der Woblfahrtspflege 6/2005

Wohngemeinschaften Blätter der Woblfahrtspflege 1/2006

Ausbildung in der Sozialen Arbeit Blätter der Wohlfahrtspflege 2/2006

Die Verortung Sozialer Arbeit Blätter der Wohlfahrtspflege 3/2006

Wirtschaft

Blätter der Wohlfahrtspflege 4/2006

Das Persönliche Budget

Blätter der Woblfahrtspflege 5/2006

Engagement als Ressource

Blätter der Woblfahrtspflege 6/2006

Soziale Arbeit und Wohnungswirtschaft

Blätter der Woblfahrtspflege 1/2007

Stiftungen

Blätter der Woblfahrtspflege 2/2007

Beratung

Blätter der Wohlfahrtspflege 3/2007

Krisendienste

Blätter der Woblfahrtspflege 4/2007

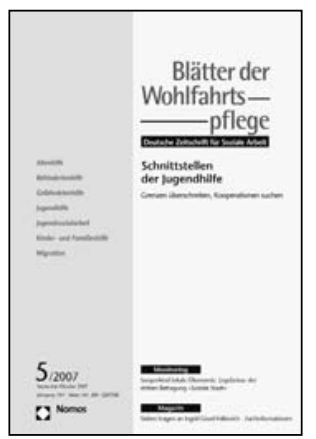

Schnittstellen der Jugendhilfe Blätter der Woblfahrtspflege 5/2007

Das Angebot wirksamer Hilfen für Menschen in schweren persönlichen Krisensituationen ist in Deutschland prekär. Gründe dafür sind unzureichende Finanzierungsgrundlagen für mobil arbeitende gemeindepsychiatrische Dienste, das Fehlen verbindlicher fachlicher Standards für psychosoziale Notfallhilfe und die geringe Bedarfsorientierung vorhandener Dienste. Die Entwicklung eines Standardangebots wirksamer psychosozialer Krisenhilfe ist eine dringliche gesundheitspolitische Aufgabe.

Der Jahresbezugspreis beträgt 58,- Euro (für Studierende und arbeitslose Bezieher auf jährlichen Nachweis 29,-Euro). Die Einzelhefte können zum Preis von 11,- Euro zuzüglich Versandkosten und Mehrwertsteuer bezogen werden bei: Nomos Verlagsgesellschaft, 76520 Baden-Baden, Telefon 07221 2104-39, Fax07221210443, E-Mail hohmann@nomos.de 